\title{
Game Utilization and Performance Following RTP From ACL Reconstruction Does not Influence a Subsequent Second ACL Injury in National Football League Players
}

\author{
Lafi S. Khalil, M.D., Kevin G. Lindsay-Rivera, M.D., Muhammad J. Abbas, B.S., \\ Sabin Shah, M.D., Marissa Tandron, M.D., Albert Ferris, B.S., and Kelechi R. Okoroha, M.D.
}

Purpose: The purpose of this study is to evaluate differences in game utilization or performance following primary anterior cruciate ligament (ACL) reconstruction between National Football League (NFL) players with and without a second ACL injury. Methods: NFL players who underwent ACL reconstruction between 2013 and 2017 were identified. Players were classified as having one injury ("tear") or having later sustained a subsequent second (reinjury or contralateral) ACL injury ("retear"). Players were excluded if they tore their ACL prior to the NFL, did not return to play (RTP), did not play the season before injury, or had concomitant injuries. Demographic characteristics, game utilization statistics, and season approximate value (SAV) performance metrics were recorded. Statistical analysis compared data after RTP from primary ACL reconstruction (seasons $+1,+2$, and +3 ) relative to the season before injury (season -1 ) between cohorts using mean differences and relative percentages. Results: Analysis included a total of 45 players, 32 in the "tear" group and 13 in the "retear" group. Demographics, level of play, and time to RTP after primary ACL reconstruction did not differ between the groups $(P>.05)$. Tear and retear groups demonstrated similar utilization and performance metrics the season prior to injury $(-1)$ and the 3 seasons following RTP (season of injury is "0"). Both groups had a similar decrease (relative percentage) in games played and started, snap counts, and SAV during the 3 seasons following RTP compared to baseline $(P>.05)$. The draft pick position was correlated with the relative percentage of games started the first season after RTP $(r=.6, P=.02)$. Conclusions: Game utilization and performance metrics following ACL reconstruction were not associated with a subsequent second ACL injury. Players with a higher draft pick position were more likely to return to the starting lineup following primary ACL reconstruction. Ultimately, player game utilization and performance following primary ACL reconstruction is not predictive of a subsequent second ACL injury. Level of Evidence: Level III, retrospective case-control study

\section{Introduction}

A nterior cruciate ligament (ACL) ruptures are one of the most prevalent knee injuries in sports with an estimated annual incidence of 200,000 nationally. ${ }^{1,2}$ National Football League (NFL) players are at an increased risk of ACL injuries compared to the general population due to the strenuous demands placed on the knee with frequent jumping, collisions, pivoting, and cutting maneuvers. ${ }^{3}$ Following ACL reconstruction, NFL players have demonstrated a high rate of return to play (RTP): $63 \%$ among linemen, ${ }^{4} 79 \%$ among wide receivers and running backs, ${ }^{5} 92 \%$ of quarterbacks, ${ }^{1}$ and $63 \%$ overall. $^{6}$ Given the prevalence of NFL athletes with a history of ACL injury, it is paramount to
Henry Ford Hospital, Department of Orthopaedic Surgery, Detroit, Michigan, U.S.A. (L.S.K., K.G.L-R., M.J.A., S.S.); Wayne State University, School of Medicine, Detroit, Michigan, U.S.A. (M.T., A.F.); and Mayo Clinic, Department of Orthopedic Surgery, Minneapolis, Minnesota, U.S.A. (K.R.O.).

The authors report the following potential conflicts of interest or sources of funding: K.R.O. has received educational support from Arthrex and Smith $\theta$ Nephew and other from Stryker and Zimmer. Full ICMJE author disclosure forms are available for this article online, as supplementary material.

Received February 15, 2021; accepted July 5, 2021.
Address correspondence to Lafi S. Khalil, M.D., Department of Orthopaedic Surgery, 2799 W. Grand Blvd., Detroit, MI 48202 U.S.A. E-mail: lskhalil@ gmail.com

(C) 2021 THE AUTHORS. Published by Elsevier Inc. on behalf of the Arthroscopy Association of North America. This is an open access article under the CC BY license (http://creativecommons.org/licenses/by/4.0/).

2666-061X/21242

https://doi.org/10.1016/j.asmr.2021.07.002 
identify factors potentially contributing to a second ACL injury in this population.

Among NFL athletes entering the NFL with a history of ACL reconstruction, there is an approximately $25 \%$ incidence of players who sustain a second ACL injury during their NFL career., ${ }^{3,7}$ When considering athletes overall following an ACL injury, prior literature suggests that the rate of retear or reinjury requiring a revision reconstruction can be up to 6 times higher in a group of athletes that had already underwent an ACL reconstruction compared with healthy controls. ${ }^{8}$ Additionally, athletes with a history of ACL reconstruction have a higher likelihood of suffering a contralateral ACL injury. ${ }^{7-9}$ The work of Paterno et al. identified the psychological toll of a primary ACL injury and subsequent reconstruction as a significant factor in increasing the risk of a second ACL tear, ${ }^{10}$ while the fear of suffering a reinjury, in turn, places a great psychological toll on athletes as well. ${ }^{1,12}$ Given the decrease in average career lifespan of NFL players to approximately 3 seasons, ${ }^{13}$ from 4.6 seasons a decade ago, ${ }^{14}$ the potential of an ACL injury to negatively affect the longevity and performance of an NFL career can be a concern for athletes and organizations. ${ }^{6,15-17}$ While the risk of a subsequent second ACL injury is certainly multifactorial, there has been limited evaluation on how the in-game utilization of players returning from primary ACL reconstruction can influence the incidence of a subsequent second ACL tear in elite NFL athletes.

There is currently a paucity of studies examining the effects of game utilization and participation following RTP from ACL reconstruction in NFL athletes as a modifiable risk factor for a subsequent second ACL injury. The purpose of this study is to evaluate differences of in game utilization or performance following primary ACL reconstruction between NFL players with and without a second ACL injury. The authors hypothesize that no differences in game utilization or performance following primary ACL reconstruction exist between NFL players with and without a subsequent ACL injury to either their ipsilateral or contralateral knee.

\section{Methods}

The authors conducted a retrospective study of ACL ruptures sustained by all NFL players from 2012/2013 season to the 2017/2018 season. Players who underwent ACL reconstruction were identified using publicly available data, such as website searches, injury reports, and team websites, using methods similar to previous studies. ${ }^{1,4,18-22}$ Additionally, players who sustained a second ACL rupture and required either a revision ACL reconstruction or a primary ACL reconstruction on the contralateral knee were identified. Injuries were confirmed using a minimum of two independent publicly available sources, which corroborate the injury. Dates of surgery were verified and cross referenced with gaps in statistical input and with team roster moves.

The control ("tear") group comprised NFL athletes who underwent a primary ACL reconstruction during the study period. The study ("retear") group comprised NFL players who underwent an initial ACL reconstruction and successful RTP, but subsequently sustained a second ACL injury to either the contralateral or ipsilateral knee. This study constitutes a case-control study of players with a primary ACL reconstruction who did and did not sustain a second ACL injury. Players were excluded from the study if any of the following conditions were met; because of missing data points, players were excluded if initial ACL injury occurred prior to entering the NFL or if they did not play the season prior to ACL injury. Additionally, a history of ACL tear prior to the NFL has been shown to negatively influence early career utilization and performance compared to healthy controls, and therefore, these players were excluded to reduce bias. ${ }^{23}$ Additionally, players were excluded if they retired or never returned to play after ACL reconstruction, or if they did not play a minimum of 8 games (half a season) during the first full season after RTP and did not play any further seasons thereafter. Other exclusion criteria included if their injury occurred as recent as the 2017/ 2018 season but their RTP game data were not yet complete enough for inclusion (minimum of 8 games/ half a season) or if they had concurrent or alternate injuries such as patellar tendon ruptures, Achilles tendon ruptures, multiligamentous knee injuries, or contralateral injuries requiring surgery (Fig 1).

Data collection methods for all players included height, weight, body mass index, age at time of injury, position, season of injury, week of injury within the season, quarter of injury within the game, starter versus bench player role (according to depth chart position) at the time of injury, time to RTP, and all reported official NFL combine performance measures and draft statistics. ${ }^{24}$ Additionally, game utilization and performance metrics were documented from football-specific statistical websites, such as pro-football-reference.com, ESPN.com, and NFL.com, and cross-referenced for validation. Game data were collected for the season prior to injury (season -1 ), the season of injury (index season), and the first 3 seasons following RTP (seasons 1,2 , and 3). The main performance metric used is the season approximate value (SAV) calculation, which is determined by pro-football-reference.com and is a numerical calculation of the relative contribution each athlete makes toward their teams' success. This calculation is based on a different formula for each position; therefore, no single formula exists, but the reported SAV is instead normalized across positions in order to 


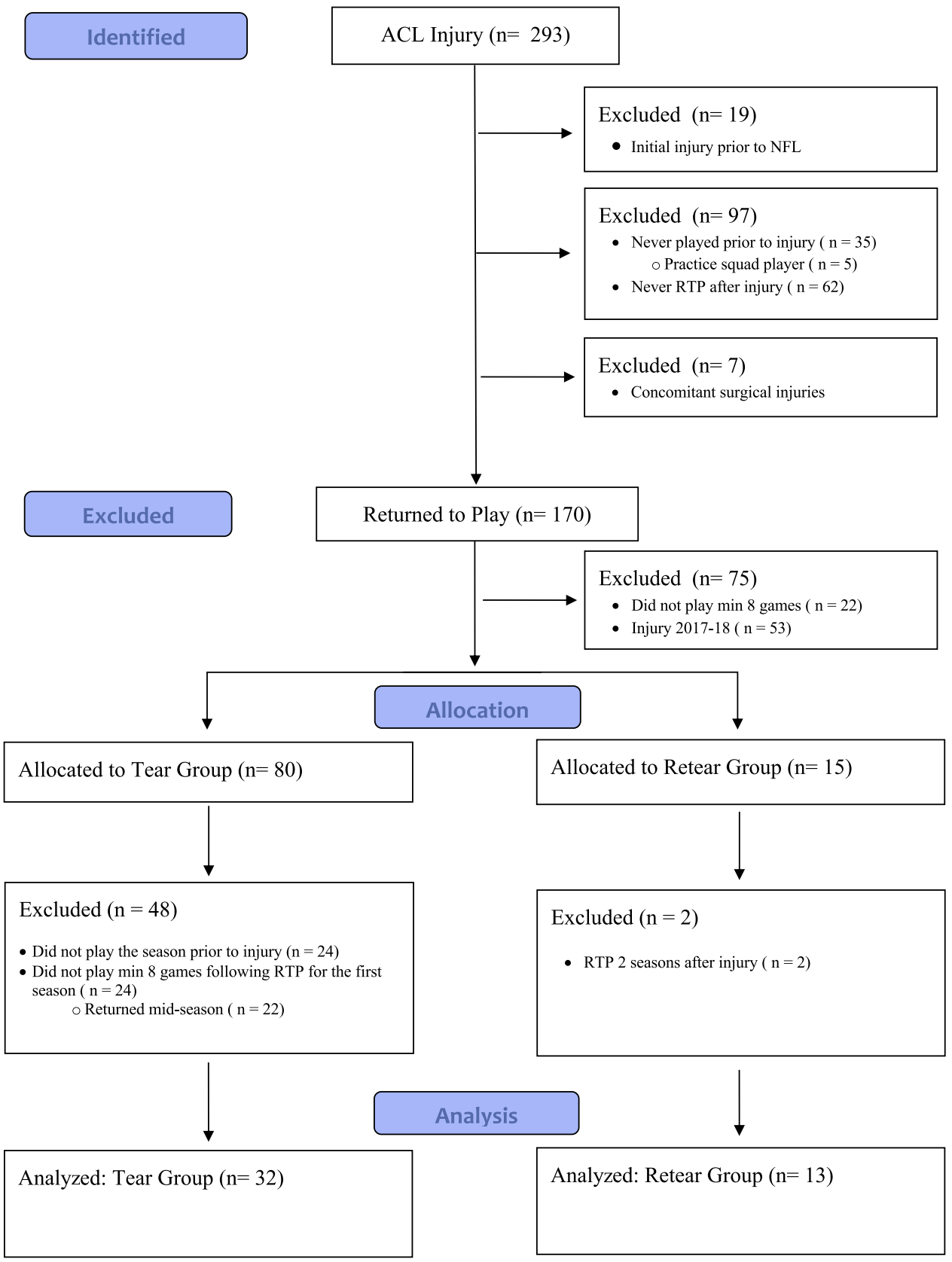

provide a standardized metric to compare athletes across positions and has been used in prior literature. The SAV is given to players at any position-weighing the relative contribution of different more commonly recorded statistics for a particular position-and generates a value that correlates to their overall helpfulness to a team in that year and has been analyzed in previous literature. ${ }^{19,25}$

Game utilization and performance variables were analyzed to evaluate changes from the baseline season before injury (season -1 ) relative to the 3 seasons following RTP. For a complete analysis, the control and study groups were compared for game utilization and performance in several manners. First, each season was compared between groups. Additionally, changes from baseline (relative to season -1 ) in seasons 1 through 3 following RTP were compared between the two groups. Lastly, relative percentages were calculated using the season prior to injury (season -1 ) as the benchmark for each individual season following RTP, similar to a prior study. ${ }^{19}$ Relative percentages for seasons 1,2 and 3 following RTP are either greater than $100 \%$ if the game utilization or performance variable exceeds that of the season prior to injury, or less than $100 \%$ if it fails to 
meet baseline. This allows an individualized and normalized comparison between groups irrespective of differences in baseline game utilization and performance. Given the short career duration of NFL athletes on average, ${ }^{13,14}$ a lengthier career assessment was not undertaken as the sample size was insufficient.

\section{Statistical Analysis}

Continuous data were reported as means \pm SD, while categorical data were reported as counts and percentages (N [\%]). For continuous variables, univariate twogroup comparisons were performed using independent 2 -sample $t$-tests if the variable was normally distributed, and Wilcoxon rank sum tests were used if the variable were non-normally distributed. For categorical variables, univariate two-group comparisons were performed using $\chi^{2}$ tests when expected cell counts were $>5$, and using Fisher's exact tests when expected cell counts were $<5$. Repeated-measures analyses were performed to see whether the performance variables changed differently over time within groups. For repeated-measures analyses, data were reported as adjusted means (standard errors) and compared between groups as well. Relative percentages were calculated for each of the 3 seasons after injury, using preinjury season 1 as the baseline. Correlation coefficients were performed between relative percentages and combine performance variables. Statistical significance was set at $P<.05$. All analyses were performed using SAS 9.4 (SAS Institute Inc., Cary, NC).

\section{Results}

\section{Demographics}

During the study period, 293 ACL injuries occurred in the NFL, of which 45 NFL players, with available game data prior to and following return from primary ACL reconstruction, met inclusion criteria (Fig 1). Thirty-two athletes sustained only primary injury and were in the "tear" group, while 13 players suffered a reinjury (ipsilateral or contralateral) and were in the "retear" group. Table 1 illustrates the demographic characteristics of both cohorts. The tear and retear groups had a mean age $( \pm S D)$ of $25.9 \pm 2.9$ and 26.6 \pm 3.3 years, and body mass index of $32.0 \pm 4.7$ and $32.7 \pm 5.3 \mathrm{~kg} / \mathrm{m}^{2}$, respectively $(P>.05)$. Numbers of seasons before injury were $3.19 \pm 2.8$ and $3.15 \pm 2.9$ for the tear and retear groups $(P>.05)$. There were no significant differences in positions played or starter versus bench player roles between groups. However, 122 of the $293(41.6 \%)$ ACL injuries initially identified, and 15 of the $45(33.3 \%)$ included in the final analysis, occurred in "speed" position players (wide receivers, linebackers, running backs, and tight ends). The timing of the first ACL injury relative to the season was evenly distributed. Of the 13 players in the retear
Table 1. Demographic and Utilization Characteristics at Time of Primary Anterior Cruciate Ligament Injury

\begin{tabular}{|c|c|c|c|}
\hline & Tear & Retear & $P$ Value \\
\hline Number of players & $32(71 \%)$ & $13(29 \%)$ & \\
\hline Age (years) & $25.9(2.9)$ & $26.6(3.3)$ & .457 \\
\hline Height (inches) & $73.9(2.6)$ & $74.5(2.9)$ & .448 \\
\hline Body mass index $\left(\mathrm{kg} / \mathrm{m}^{2}\right)$ & $32.0(4.7)$ & $32.7(5.3)$ & .673 \\
\hline Position & & & .676 \\
\hline Quarterback & $1(3 \%)$ & $2(15 \%)$ & \\
\hline Running back/Fullback & $2(6 \%)$ & $0(0 \%)$ & \\
\hline Wide receiver & $7(22 \%)$ & $1(8 \%)$ & \\
\hline Tight end & $1(3 \%)$ & $0(0 \%)$ & \\
\hline Offensive lineman & $7(22 \%)$ & $4(31 \%)$ & \\
\hline NT/DL & $5(16 \%)$ & $1(8 \%)$ & \\
\hline Defensive end & $4(13 \%)$ & $1(8 \%)$ & \\
\hline Linebacker & $2(6 \%)$ & $2(15 \%)$ & \\
\hline Cornerback/Safety & $3(9 \%)$ & $2(15 \%)$ & \\
\hline Start/Bench & & & .173 \\
\hline Starter & $15(47 \%)$ & $9(69 \%)$ & \\
\hline Bench & $17(53 \%)$ & $4(31 \%)$ & \\
\hline Time of injury & & & .969 \\
\hline Preseason & $8(25 \%)$ & $4(31 \%)$ & \\
\hline Weeks 1-4 & $4(13 \%)$ & $1(8 \%)$ & \\
\hline Weeks 5-8 & $9(28 \%)$ & $2(15 \%)$ & \\
\hline Weeks 9-12 & $3(9 \%)$ & $2(15 \%)$ & \\
\hline Weeks 13-16 & $4(1 \%)$ & $2(15 \%)$ & \\
\hline Playoffs & $2(6 \%)$ & $1(8 \%)$ & \\
\hline Offseason & $2(6 \%)$ & $1(8 \%)$ & \\
\hline SBI (seasons) & $3.19(2.82)$ & $3.15(2.91)$ & .971 \\
\hline Injured leg (first tear) & & & .141 \\
\hline Right & $20(63 \%)$ & $5(38 \%)$ & \\
\hline Left & $12(38 \%)$ & $8(62 \%)$ & \\
\hline \multicolumn{4}{|l|}{ Injured leg (second tear) } \\
\hline Ipsilateral & & $7(53.9 \%)$ & \\
\hline Contralateral & & $6(46.1 \%)$ & \\
\hline RTP primary (months) & $11.61(2.70)$ & $10.36(1.75)$ & .162 \\
\hline RTP revision (months) & & $19.10(25.92)$ & \\
\hline \multicolumn{4}{|l|}{ Combine performance } \\
\hline Draft pick & $102.2(65.1)$ & $83.3(86.7)$ & .211 \\
\hline 40 yard dash (s) & $4.76(.31)$ & $4.84(.33)$ & .48 \\
\hline Bench press (reps) & $22.2(8.5)$ & $24.2(10.2)$ & .592 \\
\hline Broad jump (in) & $113.3(7.6)$ & $110.9(10.0)$ & .472 \\
\hline Shuttle drill (s) & $4.43(.29)$ & $4.47(.30)$ & .738 \\
\hline 3 cone drill (s) & $7.20(.40)$ & $7.34(.40)$ & .404 \\
\hline Vertical (in) & $32.6(3.9)$ & $31.9(3.4)$ & .635 \\
\hline
\end{tabular}

Continuous variables are presented using mean (SD). Categorical variables are presented using frequency (percentage). NT/DL, nose tackle/defensive lineman; RTP, return to play; SBI, number of seasons before injury.

group, $7(54 \%)$ sustained ACL reruptures of the ipsilateral knee, and $6(46 \%)$ sustained an ACL injury to the contralateral knee. There was no significant difference between the tear and retear group in the average number of months to RTP after primary ACL reconstruction. The NFL combine performance metrics for players from both groups were not statistically different. Of the 293 ACL injuries discovered during the study period, it was noted that 19 players had a history of an ACL injury prior to the NFL, 35 players were rostered on a team but never played in an NFL 
Table 2. Game Utilization Trends by Season: Before and After Primary Anterior Cruciate Ligament Reconstruction

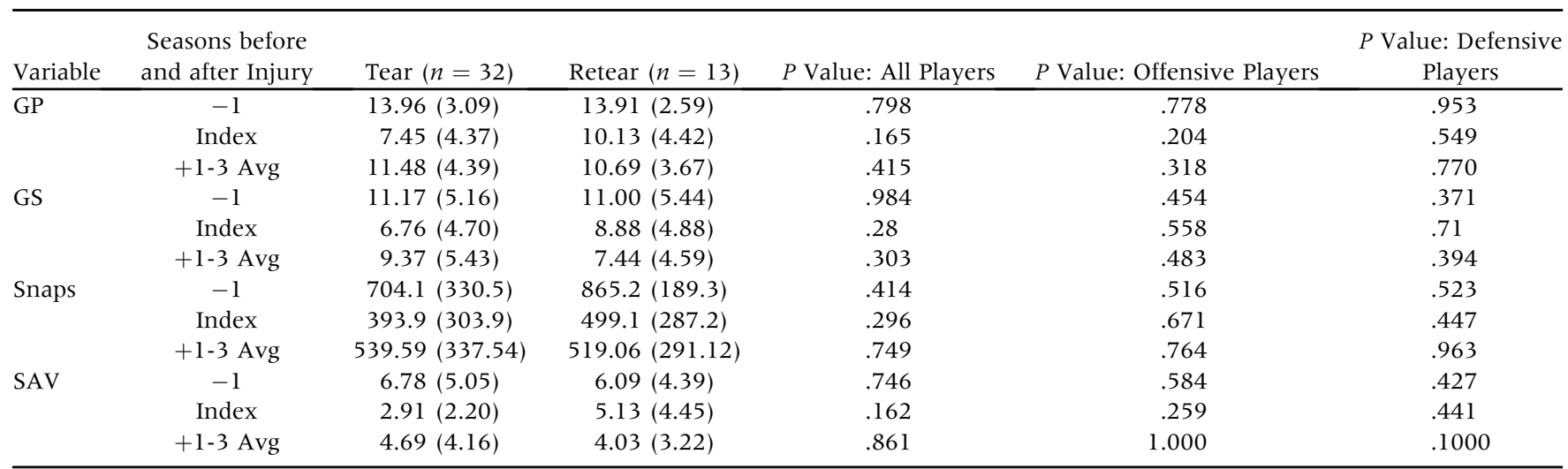

GP, games played; GS, games started; Snaps, number of snaps played; SAV, season approximate value. Seasons $+1-3$ Avg, the weighted average of each variable over the first 3 seasons following RTP. Continuous variables are presented using mean (SD).

game prior to injury, and 7 players had concomitant injuries. Therefore, the return to play rate among the remaining 232 players was $73.3 \%$ (170/232) following primary ACL reconstruction in active NFL players in the present cohort.

\section{Utilization and Performance by Season}

Table 2 illustrates utilization variables of games played (GP), games started (GS), and average snaps played per game (Snaps), as well as the standardized performance metric SAV. There were no significant differences $(P>$ $.05)$ in utilization and performance metrics between tear and retear groups the season prior to injury (season -1 ) or the 3 seasons following RTP from primary ACL reconstruction (seasons $+1,+2$, and +3 ). When cohorts were stratified by positions, no significant differences in utilization or performance metrics existed between cohorts for offensive $(P>.05)$ or defensive $(P>.05)$ players.

\section{Utilization and Performance Relative to Baseline}

Following RTP, changes in each utilization and performance variable relative to the season prior to injury (season -1 ) were calculated as adjusted mean differences for seasons $+1,+2$, and +3 (Table 3 ). Athletes in both the tear and retear group demonstrated decreased GP, GS, Snaps, and SAV during seasons $+1,+2$, and +3 , as compared to baseline (season -1 ), albeit not statistically significant $(P>.05$ for all seasons). Decreases in utilization and performance variables during each of the 3 seasons following RTP from the first ACL injury were statistically similar between the tear and retear groups $(P>.05)$. Simple logistic regression analysis demonstrated that no utilization or performance variables following primary ACL reconstruction were significantly predictive of whether the second injury occurred on the ipsilateral or contralateral knee in the retear group $(P>.05)$.

\section{Utilization and Performance Relative Percentages}

Using the season prior to injury as baseline, relative percentages were calculated for seasons $+1,+2$, and +3 following RTP from the index ACL injury for each utilization and performance variable (Fig 2). Players in the tear cohort exceeded $100 \%$ of their baseline metrics for GP, GS, Snaps, and SAV in all 3 seasons following ACL reconstruction, except for GP during season +3 $(93.2 \%)$. Players in the retear cohort failed to achieve $100 \%$ of their baseline metrics for GP, GS, Snaps, and SAV during any of the first 3 seasons following RTP from their first ACL reconstruction, except for GS during season $+1(100.6 \%)$. There were no statistically significant differences in relative percentages for any utilization or performance metric compared between tear and retear groups over the first 3 seasons following RTP $(P>.05)$.

\section{Utilization and Performance Association with Draft Position}

Table 4 displays the Pearson correlation coefficients between NFL draft combine metrics and the relative percentages for each utilization and performance metric during the first 3 seasons following RTP for all players in the tear and retear groups. A positive, mediumstrength correlation was found between the draft pick number and the relative percentage of GS 1 season after RTP (GS $+1: r=.554 ; P=.017)$. Otherwise, there were no significant correlations found between NFL combine performance and utilization and performance metrics following RTP from primary ACL reconstruction.

\section{Discussion}

Among NFL players who played at least 1 season in the NFL prior to their primary ACL reconstruction, there were no significant differences in game utilization and performance upon RTP between players who never 
Table 3. Adjusted Mean Differences Following RTP Relative to the Season Before Injury

\begin{tabular}{|c|c|c|c|c|}
\hline Variable & $\begin{array}{l}\text { Season } \\
\text { after RTP }\end{array}$ & Tear & Retear & $P$ Value \\
\hline \multirow[t]{4}{*}{$\overline{\mathrm{GP}}$} & +1 & $-1.93(1.17)$ & $-2.45(1.92)$ & .653 \\
\hline & +2 & $-2.5(1.24)$ & $-4.21(1.96)$ & .461 \\
\hline & +3 & $-3.3(1.37)$ & $-3.74(2.28)$ & .872 \\
\hline & $P$ value* & $>.05$ & $>.05$ & \\
\hline \multirow[t]{4}{*}{ GS } & +1 & $-1.01(1.58)$ & $-4.5(2.42)$ & .265 \\
\hline & +2 & $-1.34(1.7)$ & $-1.14(2.67)$ & .502 \\
\hline & +3 & $-1.11(1.8)$ & $-2(2.96)$ & .363 \\
\hline & $P$ value ${ }^{*}$ & $>.05$ & $>.05$ & \\
\hline \multirow[t]{4}{*}{ Snaps } & +1 & $-133.79(91.08)$ & $-306.28(183.7)$ & .467 \\
\hline & +2 & $-183.39(96.74)$ & $-392.5(183.7)$ & .153 \\
\hline & +3 & $-182.26(109.96)$ & $-315(201.23)$ & .585 \\
\hline & $P$ value ${ }^{*}$ & $>.05$ & $>.05$ & \\
\hline \multirow[t]{4}{*}{ SAV } & +1 & $-1.68(1.19)$ & $-1.84(1.89)$ & .929 \\
\hline & +2 & $-1.81(1.23)$ & $-2.59(1.97)$ & .268 \\
\hline & +3 & $-1.33(1.37)$ & $-.23(2.18)$ & .591 \\
\hline & $P$ value $*$ & $>.05$ & $>.05$ & \\
\hline
\end{tabular}

GP, games played; GS, games started; RTP, return to play; SAV, season approximate value; Snaps, number of snaps played. Adjusted mean difference (standard error) of each variable during seasons 1-3 relative to the season prior to injury (season -1 ). Adjusted mean differences for seasons $+1,+2$, and +3 are compared relative to the season prior to injury $(-1)$

*Within each group, with all 3 seasons having nonsignificant $P$ values.

${ }^{\ddagger}$ Adjusted mean differences compared between groups.

sustained a subsequent second ACL tear (tear players) and those who did (retear players). Although tear players returned to baseline game utilization and performance following ACL reconstruction, averaging greater than $100 \%$ relative percentages during the first 3 seasons, this was not statistically different than retear players who predominantly failed to achieve baseline markers. This finding highlights that game utilization and performance, as surrogate measures of overall athletic demand following primary ACL reconstruction, are not predictive of another ACL injury. Interestingly, players with a higher NFL draft pick position demonstrated a significant correlation with the relative percentage of games started following RTP from primary ACL reconstruction. Overall, $73.3 \%$ of NFL players were able to RTP following primary ACL reconstruction.

The present study sought to determine whether game utilization and performance following RTP from primary ACL reconstruction was a significant predictive factor among NFL players who subsequently sustained a second ACL injury compared to those who did not. The presented results demonstrated no significant differences in snaps, GS, or GP, and the amalgamation statistic of value (SAV) to team. When analyzing the data based on subgroups, such as position of the player, a similar lack of significance was seen. These results echo the findings of Okoroha et al., who evaluated time to RTP following primary ACL reconstruction and found it not to be a risk factor of sustaining a reinjury. ${ }^{26}$ Likewise, Cinque et al. compared NFL linemen who RTP following primary ACL reconstruction to a healthy, matched control group, demonstrating no significant differences in game performance or career longevity between the two cohorts. ${ }^{4}$ Despite the encouraging findings following ACL reconstruction in the previously mentioned studies, neither evaluated game utilization and performance as a risk factor for ACL reinjury.

The higher risk of reinjury in NFL athletes who have had a prior ACL injury is well established in the literature. $^{3,8}$ The work of Dodson et al. demonstrates that nearly one-fifth $(18.3 \%)$ of ACL injuries sustained were in fact the players' second ACL injury, with $12.3 \%$ retears in players that had previously undergone an ipsilateral ACL reconstruction and $7.3 \%$ tears to the contralateral knee. ${ }^{3}$ In a review of NFL combine participants with a prior ACL injury, Connor et al. found a $25 \%$ incidence of a subsequent second ACL injury (12 ipsilateral and 14 contralateral) during their NFL career, which was significantly greater than the incidence of primary ACL injury among a healthy, matched control cohort $(18 / 200=9 \% ; P<.001){ }^{7}$ The results from the present study demonstrated that from a cohort of NFL athletes who played in NFL games prior to their first ACL reconstruction and successfully returned to play for at least half of an NFL season, 28.9\% (13/45) sustained a second ACL injury, with approximately equal distribution of reinjury and contralateral injury (54\% vs $46 \%$ ). Although these results show a greater incidence of subsequent injury than prior literature, it is likely secondary to the stringent inclusion criteria applied. This was done intentionally to limit potentially confounding factors introduced by heterogenous data of players with no prior NFL experience or significant RTP time after injury.

In evaluating player factors contributing to injury, Dodson et al. determined that most players who sustained a second ACL injury were in so-called "speed" positions, including wide receivers, linebackers, running backs, and tight ends. ${ }^{3}$ In fact, most NFLcaliber athletes who sustain an ACL tear do so through a noncontact mechanism. Retrospective video analyses revealed that $72 \%$ of ACL injuries in the NFL occur with a pivoting motion, as is often exhibited by a "speed" player who places a large valgus moment on the affected extremity at the knee. ${ }^{27}$ In the present study, 122 of the 293 (41.6\%) ACL injuries initially identified, and 15 of the $45(33.3 \%)$ included in the final analysis, occurred in speed position players. As this biomechanical, noncontact pivoting mechanism may be related to fatigue from overuse and neuromuscular deconditioning, ${ }^{28,29}$ it is prudent to continue evaluating whether "load management" strategies are effective in reducing the risk of reinjury. ${ }^{30,31}$ Given the prevalence 
A

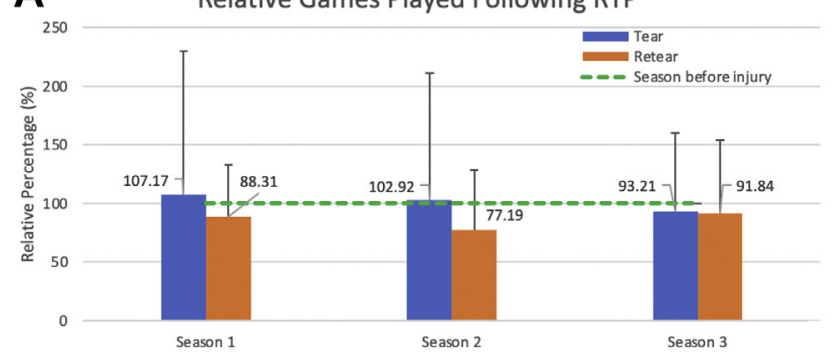

B

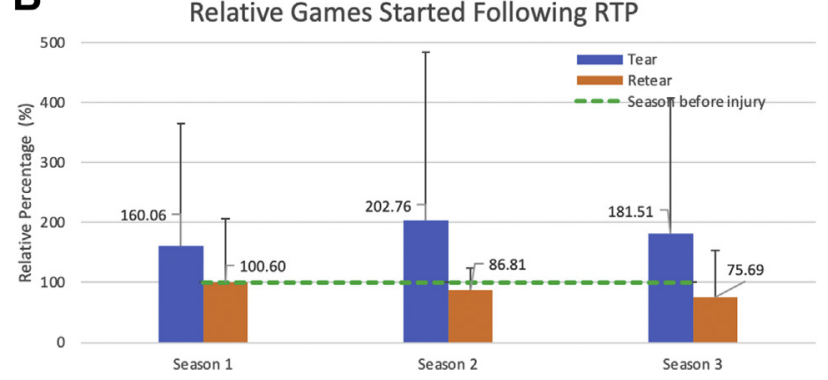

C

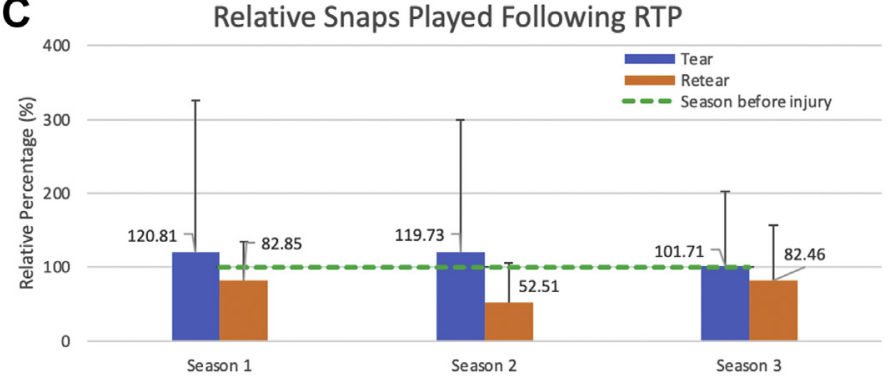

D

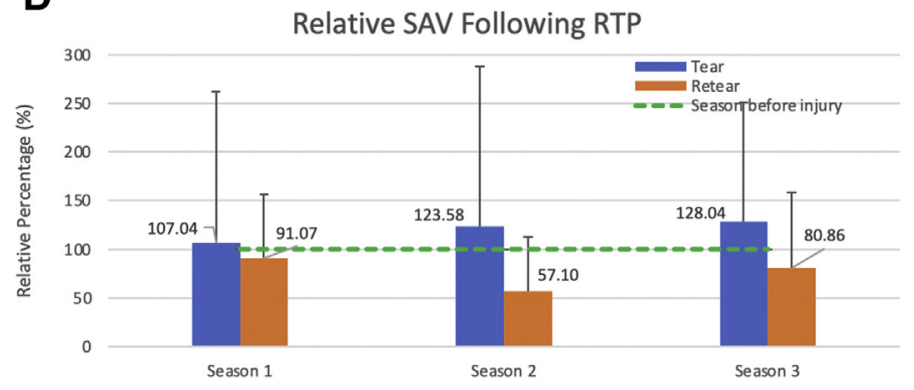

Fig 2. Relative percentage of games played (A), games started (B), snaps played (C), and season approximate value (SAV) (D) during the first three seasons following return to play (RTP) from anterior cruciate ligament reconstruction among tear and retear players. Preinjury baseline is represented by the dotted horizontal line $(100 \%)$ across all variables, which corresponds to the season prior to injury. Relative percentages below this line indicate that the post-index variable was less than baseline, while values above this line indicate an increase in post-index variable relative to baseline. *No significant differences $(P<.05)$ exist between tear and retear groups.

of ACL injury in NFL players and the high degree of collisions that place relatively large stresses on the reconstructed knee, ${ }^{11,32-34}$ there has been a concerted effort to address a player's return to full-time duty and determine appropriate game utilization while minimizing reinjury. ${ }^{1,4,26,35,36}$

The present results did indicate that players picked with higher draft positions were significantly more likely to start in games the season after RTP from ACL reconstruction. This phenomenon parallels findings from a prior study by Okoroha et al., which investigated RTP following revision ACL reconstruction and determined that NFL athletes with higher draft positioning or greater NFL experience prior to revision surgery were more likely to RTP. ${ }^{35}$ Daruwalla et al. similarly demonstrated that college players higher in the depth

Table 4. Pearson Correlations Between Relative Percentages Per Season Following Return to Play with National Football League Combine and Draft Performance

\begin{tabular}{lcccccccc}
\hline Relative $\%$ & Season & Draft Pick & 40-Yard Dash & Bench Press & Broad Jump & Shuttle Drill & 3-Cone Drill & Vertical Jump \\
\hline GP & +1 & -.05905 & .18312 & -.05817 & -.13112 & .05257 & -.02138 & -.08522 \\
& +2 & -.2902 & .16458 & -.09883 & -.20308 & -.0521 & -.00786 & -.07397 \\
& +3 & -.24005 & .16444 & -.04761 & -.18193 & .00902 & .05111 & -.16958 \\
GS & +1 & .55365 & -.01877 & -.06586 & .03925 & -.20062 & -.26434 & .0818 \\
& +2 & .08278 & -.14673 & -.08272 & -.08949 & -.22439 & -.3256 & .19003 \\
& +3 & .4081 & -.13769 & .00555 & -.0515 & -.39057 & -.3389 & .1771 \\
\multirow{2}{*}{ Snaps } & +1 & .23361 & .1568 & -.11643 & -.11833 & .03247 & -.07479 & -.07056 \\
& +2 & -.0865 & .05237 & -.31587 & -.13145 & -.07008 & -.07561 & .02423 \\
& +3 & -.32457 & .18144 & -.08385 & -.16872 & -.09243 & -.09267 & -.15369 \\
\multirow{2}{*}{ SAV } & +1 & -.19553 & .1847 & -.00445 & -.18584 & -.02145 & -.08167 & -.06645 \\
& +2 & -.08737 & .04801 & -.1055 & -.10953 & -.12522 & -.14765 & .04431 \\
& +3 & .1457 & -.09438 & -.22935 & -.07532 & -.34645 & -.27418 & .18729
\end{tabular}

National Football League combine and draft performance correlations with the relative percentages of games played (GP), games started (GS), snaps played, and season approximate value (SAV) during seasons 1 through 3 following return to play from primary anterior cruciate ligament reconstruction relative to the season before injury (season -1 ). Pearson correlation coefficients expressed as Prob $>|r|$ under H0: Rho $=0$. Significant values are noted in bold; $P<.05$. 
chart were more likely to RTP following ACL reconstruction. ${ }^{37}$ It is worth noting that the current study excluded players without prior NFL experience, and both tear and retear groups had similar NFL experience prior to their first ACL injury. One strength of this study is the novelty in comparing a cohort of athletes who sustained their index ACL injury while in the NFL, thereby enabling objective evaluation of their game utilization and performance upon RTP relative to their NFL preinjury baseline utilization and performance, compared with those later sustaining a second ACL injury. The finding of draft pick position influencing rates of RTP and GS highlights the competitive nature of an NFL career and the potential for future studies to evaluate whether draft pick position and game performance may, in fact, be greater predictors of career longevity than a history of ACL reconstruction or subsequent reinjury.

\section{Limitations}

This study, as with other retrospective studies, has limitations in the form of potential bias and confounding variables impacting the results of the study. Largely, these biases were introduced in the data collection as an Internet-based review of statistics websites. Nevertheless, the methodology closely resembled that of prior studies. ${ }^{1,4,18-22}$ Similarly, these data and conclusions cannot be applied to a more general population of athletes or for other levels of play in American football such as college, high school, or youth football, as the present cohorts specifically sustained their primary injury while in the NFL. Furthermore, the classification and grading of injuries, and other concomitant injuries, such as meniscal and cartilage damage, was not possible due to the lack of availability of official medical records and imaging for review. Likewise, surgical technique, graft selection, fixation methods, and postoperative rehabilitation protocols were not standardized. As the NFL is a competitive league and game decisions are made by coaching staff and roster composition, some factors may confound game utilization and performance for which we cannot account. Because of the lack of publicly reported statistical data prior to the study period (2012), the sample size is too small to independently analyze players with ipsilateral and contralateral reinjuries; therefore, the current study is unable to predict the risk of ipsilateral or contralateral reinjury independently. A power analysis was not conducted, as all players with available statistics since 2012 were eligible for inclusion. Future studies with larger sample sizes are required to determine these differences. Exclusion of NFL athletes with a history of ACL injury prior to entering the NFL greatly limited the sample size of the present study, but it prevented the analysis of heterogenous data as all included players' game data were compared chronologically relative to the index season.

\section{Conclusion}

Game utilization and performance metrics following ACL reconstruction were not associated with a subsequent second ACL injury. Players with a higher draft pick position were more likely to return to the starting lineup following primary ACL reconstruction. Ultimately, player game utilization and performance following primary ACL reconstruction are not predictive of a subsequent second ACL injury.

\section{References}

1. Erickson BJ, Harris JD, Heninger JR, et al. Performance and return-to-sport after ACL reconstruction in NFL quarterbacks. Orthopedics 2014;37:e728-e734.

2. DeHaven KE, Lintner DM. Athletic injuries: Comparison by age, sport, and gender. Am J Sports Med 1986;14: 218-224.

3. Dodson CC, Secrist ES, Bhat SB, Woods DP, Deluca PF. Anterior cruciate ligament injuries in National Football League athletes from 2010 to 2013: A descriptive epidemiology study. Orthop J Sports Med 2016;4: 2325967116631949.

4. Cinque ME, Hannon CP, Bohl DD, et al. Return to sport and performance after anterior cruciate ligament reconstruction in National Football League linemen. Orthop J Sports Med 2017;5:2325967117711681.

5. Carey JL, Huffman GR, Parekh SG, Sennett BJ. Outcomes of anterior cruciate ligament injuries to running backs and wide receivers in the National Football League. Am J Sports Med 2006;34:1911-1917.

6. Shah VM, Andrews JR, Fleisig GS, McMichael CS, Lemak LJ. Return to play after anterior cruciate ligament reconstruction in National Football League athletes. Am J Sports Med 2010;38:2233-2239.

7. Stuhlman CR, Owens CJ, Samuelson EM, et al. Recurrent anterior cruciate ligament tears in the National Football League: A case-control study. Orthop J Sports Med 2019;7: 2325967119891413.

8. Paterno MV, Rauh MJ, Schmitt LC, Ford KR, Hewett TE. Incidence of second ACL injuries 2 years after primary ACL reconstruction and return to sport. Am J Sports Med 2014; 42:1567-1573.

9. Paterno MV, Rauh MJ, Schmitt LC, Ford KR, Hewett TE. Incidence of contralateral and ipsilateral anterior cruciate ligament (ACL) injury after primary ACL reconstruction and return to sport. Clin J Sport Med 2012;22:116-121.

10. Paterno MV, Flynn K, Thomas S, Schmitt LC. Self-reported fear predicts functional performance and second ACL injury after ACL reconstruction and return to sport: A pilot study. Sports Health 2018;10:228-233.

11. Morrey MA, Stuart MJ, Smith AM, Wiese-Bjornstal DM. A longitudinal examination of athletes' emotional and cognitive responses to anterior cruciate ligament injury. Clin J Sport Med 1999;9:63-69.

12. Kvist J, Ek A, Sporrstedt K, Good L. Fear of re-injury: a hindrance for returning to sports after anterior cruciate 
ligament reconstruction. Knee Surg Sports Traumatol Arthrosc 2005;13:393-397.

13. Arthur R. The shrinking shelf life of NFL players. The Wall Street Journal Feb 29, 2016, https://www.wsj.com/articles/ the-shrinking-shelf-life-of-nfl-players-1456694959.

14. Davis ME, Lombardi VP, Troxel RK. Injuries in National Football League prospects $\&$ longevity by position in the NFL: 843. [abstract]. Med Sci Sports Ex 2009;41:97.

15. Carmont MR, Ennis O, Rees D. Return to play after anterior cruciate ligament reconstruction in National Football League athletes" by Shah et al. Am J Sports Med 201 1;39:NP3.

16. Busfield BT, Kharrazi FD, Starkey C, Lombardo SJ, Seegmiller J. Performance outcomes of anterior cruciate ligament reconstruction in the National Basketball Association. Arthroscopy 2009;25:825-830.

17. Ardern CL, Webster KE, Taylor NF, Feller JA. Return to sport following anterior cruciate ligament reconstruction surgery: A systematic review and meta-analysis of the state of play. Br J Sports Med 2011;45:596-606.

18. Amin NH, Old AB, Tabb LP, Garg R, Toossi N, Cerynik DL. Performance outcomes after repair of complete Achilles tendon ruptures in national basketball association players. Am J Sports Med 2013;41:1864-1868.

19. Trofa DP, Miller JC, Jang ES, Woode DR, Greisberg JK, Vosseller JT. Professional athletes' return to play and performance after operative repair of an Achilles tendon rupture. Am J Sports Med 2017;45:2864-2871.

20. Mehran N, Williams PN, Keller RA, Khalil LS, Lombardo SJ, Kharrazi FD. Athletic performance at the National Basketball Association combine after anterior cruciate ligament reconstruction. Orthop J Sports Med 2016;4:2325967116648083.

21. Keller RA, Mehran N, Khalil LS, Ahmad CS, ElAttrache N. Relative individual workload changes may be a risk factor for rerupture of ulnar collateral ligament reconstruction. J Shoulder Elbow Surg 2017;26:369-375.

22. Keller RA, Mehran N, Marshall NE, et al. Major League pitching workload after primary ulnar collateral ligament reconstruction and risk for revision surgery. J Shoulder Elbow Surg 2017;26:288-294.

23. Provencher MT, Bradley JP, Chahla J, et al. A history of anterior cruciate ligament reconstruction at the National Football League combine results in inferior early National Football League career participation. Arthroscopy 2018;34: 2446-2453.

24. 2020 NFL Combine Results. Pro Football Reference: Sports Reference LLC. Vol. 2021, https://www.profootball-reference.com/draft/2020-combine.htm 2020.

25. Khalil LS, Jildeh TR, Abbas MJ, et al. Career longevity and performance after shoulder instability in national football league athletes. Arthroscopy 2021;37:1437-1445.

26. Okoroha KR, Fidai MS, Tramer JS, et al. Length of time between anterior cruciate ligament reconstruction and return to sport does not predict need for revision surgery in National Football League players. Arthroscopy 2019;35: 158-162.

27. Johnston JT, Mandelbaum BR, Schub D, et al. Video analysis of anterior cruciate ligament tears in professional American Football athletes. Am J Sports Med 2018;46: 862-868

28. Fidai MS, Okoroha KR, Meldau J, et al. Fatigue increases dynamic knee valgus in youth athletes: Results from a field-based drop-jump test. Arthroscopy 2020;36:214-222 e212.

29. Hunnicutt JL, Jayanthi NA, Labib SA. Editorial Commentary: Considering fatigue when assessing athletes for dynamic knee valgus: Is this the next big step in identifying anterior cruciate ligament injury risk? Arthroscopy 2020;36:223-224.

30. Bourne MN, Webster KE, Hewett TE. Is fatigue a risk factor for anterior cruciate ligament rupture? Sports Med 2019:49:1629-1635.

31. Zaffagnini S, Grassi A, Serra M, Marcacci M. Return to sport after ACL reconstruction: how, when and why? A narrative review of current evidence. Joints 2015;3: 25-30.

32. Edwards T, Spiteri T, Piggott B, Haff GG, Joyce C. A narrative review of the physical demands and injury incidence in American Football: Application of current knowledge and practices in workload management. Sports Med 2018:48:45-55.

33. Fullagar HHK, McCunn R, Murray A. Updated review of the applied physiology of American college football: Physical demands, strength and conditioning, nutrition, and injury characteristics of America's favorite game. Int J Sports Physiol Perform 2017;12:1396-1403.

34. Goodman AD, Raducha JE, DeFroda SF, Gil JA, Owens BD. Shoulder and elbow injuries in NCAA football players, 2009-2010 through 2013-2014. Phys Sportsmed 2019;47:323-328.

35. Okoroha KR, Kadri O, Keller RA, Marshall N, Cizmic Z, Moutzouros V. Return to play after revision anterior cruciate ligament reconstruction in National Football League players. Orthop J Sports Med 2017;5: 2325967117698788.

36. Mihoces G. Adrian Peterson leads way in ACL recovery in NFL. Vol 2020. USA TODAY Sports Dec 6, 2012, https:// WWW.usatoday.com/story/sports/nfl/2012/12/06/aclinjuries-adrian-peterson-nfl-minnesota-vikings / 175 $2355 \%$

37. Daruwalla JH, Greis PE, Hancock R, Group ASPC, Xerogeanes JW. Rates and determinants of return to play after anterior cruciate ligament reconstruction in NCAA Division 1 College Football athletes: A study of the ACC, SEC, and PAC-12 Conferences. Orthop J Sports Med 2014;2: 2325967114543901. 